\title{
SOME PREDICTIONS FOR AN IMPROVED FERMION ACTION ON THE LATTICE
}

\author{
Herbert W. HAMBER and Chi Min WU \\ The Institute for Advanced Study, Princeton, NJ 08540, USA
}

Received 30 September 1983

\begin{abstract}
We propose an improved fermion action on the lattice by adding a next nearest neightbor interaction term to the Wilson action. The proposed action is expected to approach the continuum limit more rapidly. Using the improved action, the predictions for the critical value of the hopping parameter at weak and strong coupling are given. The relationship between quark masses on the lattice and in the continuum is also discussed.
\end{abstract}

1. Introduction. Recently numerical studies of lattice gauge theories in the form of numerical simulations have been pursued in an attempt to compute non-perturbative quantities such as mass spectra [1]. It is known that the properties of the continuum euclidean quantum field theory are supposed to be recovered in the limit when the lattice spacing $a$ goes to zero. Finite size and finite bare coupling effects are two types of problems that affect these computations and which have to be seriously considered. One possible approach to the question of whether the theory is really physically relevant is to try to investigate whether theoretically motivated improvements also lead to improvements in the quality of the numerical results.

As first suggested by Wilson and by Symanzik [2] in the framework of $\varphi_{4}^{4}$, one possible approach is to systematically construct a lattice action such that the cutoff dependence of physical observables is reduced and the continuum scaling behaviour is approached more rapidly. Recently the improvement program was studied in the context of the non-linear sigma model in two dimensions by Symanzik and collaborators [3], by Martinelli et al. [4], and in four-dimensional pure Yang-Mills field by Weisz [5] and by Curci et al. [6] .

In this paper we propose an improved fermion action on the lattice by adding a next nearest neighbor interaction term to the Wilson fermion action [7], and present some analytical predictions for this new action.

2. Improved action and mass renormalization. We propose an action of the following form

$S=S_{\mathrm{G}}+S_{\mathrm{F}}$

with

$$
\begin{aligned}
S_{\mathrm{G}} & =\frac{1}{g^{2}} \sum_{n, \mu<\nu} \operatorname{tr}\left[U_{n, \mu} U_{n+\mu, \nu} U_{n+\nu, \mu}^{+} U_{n, \nu}^{+}+\text {h.c. }-2\right], \\
S_{\mathrm{F}} & =k \sum_{n, \mu}\left[\bar{\psi}_{n}\left(r-\gamma_{\mu}\right) U_{n, \mu} \psi_{n+\mu}+\bar{\psi}_{n+\mu}\left(r+\gamma_{\mu}\right) U_{n, \mu}^{+} \psi_{n}\right] \\
& \sum_{n, \mu}\left[\bar{\psi}_{n}\left(C-D \gamma_{\mu}\right) U_{n, \mu} U_{n+\mu, \mu} \psi_{n+2 \mu}+\bar{\psi}_{n+2 \mu}\left(C+D \gamma_{\mu}\right) U_{n+\mu, \mu}^{+} U_{n, \mu}^{+} \psi_{n}\right]-\sum_{n} \bar{\psi}_{n} \psi_{n} .
\end{aligned}
$$

As can be seen, we have added a next nearest neighbor interaction term to the Wilson fermion action. The coefficients $C$ and $D$ will be determined later. 


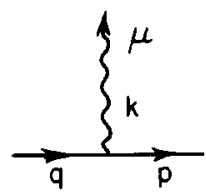

(a)

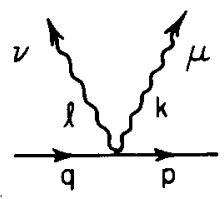

(b)
Fig. 1. (a), (b) Fermion-gluon vertices appearing in the improved fermion action.

In eq. (2.2) the gauge group is $\mathrm{SU}(N)$ and the $U_{n, \mu}$ 's are $N$ by $N$ complex matrices belonging to the group $\mathrm{SU}(N)$. We have set the lattice spacing $a=1$ and

$U_{n, \mu}=\exp \left(\mathrm{i} g A_{n, \mu}\right), \quad A_{n, \mu}=\sum_{a} A_{n \mu}^{a} t^{a}$.

The $t^{a}$ 's are the generators of SU(N) and are normalized to tr $t^{a} t^{b}=\frac{1}{2} \delta^{a b}$. The hopping parameter $k$ is related to the fermion mass.

In the usual way, we get the Feynman rules for the gluon and fermion propagators

$D_{\mu \nu}(q)=\left[1 / S^{2}(q)\right]\left[\delta_{\mu \nu}-(1-\lambda) S_{\mu}(q) S_{\nu}(q) / S^{2}(q)\right]$,

$S_{\mathrm{F}}^{-1}(p)=1-2 k \sum_{\mu}\left(r \cos p_{\mu}-\mathrm{i} \gamma_{\mu} \sin p_{\mu}\right)-2 \sum_{\mu}\left(C \cos 2 p_{\mu}-\mathrm{i} D \gamma_{\mu} \sin 2 p_{\mu}\right)$

and for the two fermion-gluon vertices (see fig. $1 \mathrm{a}, \mathrm{b}$ )

$$
V_{\mu}^{(3)}(p, q)=2 g\left[k r \sin \frac{1}{2}(p+q)_{\mu}+\mathrm{i} k \gamma_{\mu} \cos \frac{1}{2}(p+q)_{\mu}\right.
$$$$
\left.+2 C \sin (p+q)_{\mu} \cos \frac{1}{2}(p-q)_{\mu}+2 \mathrm{i} D \gamma_{\mu} \cos (p+q)_{\mu} \cos \frac{1}{2}(p-q)_{\mu}\right] t^{a},
$$

$V_{\mu \nu}^{(4)}(p, q)=-2 k g^{2} \delta_{\mu \nu}\left[r \cos \frac{1}{2}(p+q)_{\mu}-\mathrm{i} \gamma_{\mu} \sin \frac{1}{2}(p+q)_{\mu}\right] t^{a} t^{b}$

$$
-2 g^{2} \delta_{\mu \nu}\left[4 C \cos \frac{1}{2} k_{\mu} \cos (p+q)_{\mu} \cos \frac{1}{2} l_{\mu}-4 \mathrm{i} D \gamma_{\mu} \cos \frac{1}{2} k_{\mu} \sin (p+q)_{\mu} \cos \frac{1}{2} l_{\mu}\right] t^{a} t^{b},
$$

where $S_{\mu}(q)=2 \sin \frac{1}{2} q_{\mu}$ and $S^{2}(q)=\Sigma_{\mu} S_{\mu}^{2}(q)$. We shall work in the Feynman gauge where $\lambda=1$. As $p \rightarrow 0$, we expand $S_{\mathrm{F}}^{-1}(p)$ in powers of $p$.

$S_{\mathrm{F}}^{-1}(p)=1-8 k r-8 C+\mathrm{i} \sum_{\mu} \gamma_{\mu} p_{\mu}(2 k+4 D)+\sum_{\mu} p_{\mu}^{2}(k r+4 C)+\mathrm{i} \sum_{\mu} \gamma_{\mu} p_{\mu}^{3}\left(-\frac{1}{3} k-\frac{8}{3} D\right)+\ldots$

Let us take the coefficient for the term $\mathrm{O}\left(p^{2}\right)$ to be zero, i.e.

$C=-\frac{1}{4} k r, \quad D=C / r$.

In this way, not only the inverse propagator $S_{\mathrm{F}}^{-1}(p)$ but also the vertices approach the continuum limit more rapidly.

Let us now consider the fermion mass renormalization effects for the new action. The lowest order diagrams are given in fig. 2(a) and (b) and their contributions are

$$
\Sigma^{(\mathrm{a})}(p)=\sum_{\mu, \nu} \int_{q} V_{\mu}^{(3)}\left(p-\frac{1}{2} q\right) S_{\mathrm{F}}(p-q) D_{\mu \nu}(q) V_{\nu}^{(3)}\left(p-\frac{1}{2} q\right), \quad \Sigma^{(\mathrm{b})}(p)=\frac{1}{2} \sum_{\mu, \nu} \int_{q} V_{\mu \nu}^{(4)}(p, q) D_{\mu \nu}(q) \delta_{\mu \nu},
$$




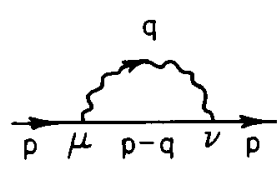

(a)

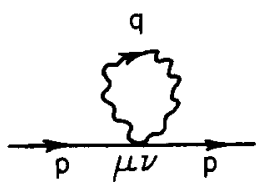

(b)
Fig. 2. (a), (b) Diagrams contributing to lowest order to the fermion self-energy.

where we have set for brevity

$\int_{q}=\prod_{\mu=1}^{4} \int_{-\pi}^{+\pi} \frac{\mathrm{d} q_{\mu}}{2 \pi}$

We now perform a Taylor series expansion around $p_{\mu}=0$ for $\Sigma^{(a)}$ and $\Sigma^{(b)}$, and parameterize the first two terms in the expansion as

$\Sigma^{(\mathrm{a})}(p)=-k\left[\delta m^{(\mathrm{a})}+\frac{1}{3} \mathrm{i} \sum_{\mu} \gamma_{\mu} p_{\mu}^{3} A^{(\mathrm{a})}+B^{(\mathrm{a})}\left(\mathrm{i} \not p+m+\frac{1}{3} \mathrm{i} \sum_{\mu} \gamma_{\mu} p_{\mu}^{3}\right)\right]$,

with $m=(1-6 k r) / k$. A similar expansion can also be done for $\Sigma^{(\mathrm{b})}$. We thus obtain the modified fermion propagator

$$
\begin{aligned}
& S^{\prime-1}(p)=S_{\mathrm{F}}^{-1}(p)-\Sigma^{(\mathrm{a})}(p)-\Sigma^{(\mathrm{b})}(p) \\
& \quad=k\left[1+B^{(\mathrm{a})}+B^{(\mathrm{b})}\right]\left(\not \not p+m+\delta m^{(\mathrm{a})}+\delta m^{(\mathrm{b})}+\frac{1}{3} \mathrm{i} \sum_{\mu} \gamma_{\mu} p_{\mu}^{3}\left(1+A^{(\mathrm{a})}+A^{(\mathrm{b})}\right)\right) .
\end{aligned}
$$

The term $k\left[1+B^{(a)}+B^{(b)}\right]$ can be absorbed into the wave function renormalization. Using the Feynman rules of eqs. (2.4)-(2.7), we get $\delta m^{(a)}$ and $\delta m^{(b)}$ at $k=1 / 6 r$ (which corresponds to vanishing quark mass, $m=0$ )

$\delta m^{(\mathrm{a})}=-\frac{1}{4}\left(\frac{N^{2}-1}{2 N}\right) g^{2} r \int_{q} \frac{\Sigma_{\alpha} \sin ^{4} \frac{1}{2} q_{\alpha}\left[2 X_{\alpha}-\Sigma_{\mu} X_{\mu}+r^{2} \Sigma_{\mu} \sin ^{6} \frac{1}{2}\right.}{\left[r^{2}\left(\Sigma_{\alpha} \sin ^{4} \frac{1}{2} q_{\alpha}\right)^{2}+\Sigma_{\alpha} X_{\alpha} \sin ^{2} \frac{1}{2} q_{\alpha}\right] \Sigma_{\rho} \sin ^{2} \frac{1}{2} q_{\rho}}$,

$\delta m^{(\mathrm{b})}=\frac{1}{4}\left[\left(N^{2}-1\right) / 2 N\right] g^{2} r$,

where

$X_{\alpha}=\cos ^{2} \frac{1}{2} q_{\alpha}\left(\frac{1}{2}+\sin ^{2} \frac{1}{2} q_{\alpha}\right)^{2}$.

Doing the integrals numerically we find also

$\delta m^{(a)}=-0.0134\left[\left(N^{2}-1\right) / 2 N\right] g^{2} r$,

so that $\delta m^{(\mathrm{b})}$ is much larger than $\delta m^{(\mathrm{a})}$. Therefore, we neglect the contribution of $\delta m^{(\mathrm{a})}$. Thus, from

$m+\delta m^{(\mathbf{b})}=0$,

we obtain $k_{\mathrm{c}}$ in a weak coupling expansion

$k_{\mathrm{c}}=(1 / 6 r)\left[1+\frac{1}{24}\left[\left(N^{2}-1\right) / 2 N\right] g^{2}+O\left(g^{4}\right)\right]=(1 / 6 r)\left[1+\frac{1}{18} g^{2}+O\left(g^{4}\right)\right], \quad$ for SU(3) 
Next, we will calculate the meson propagators to find a singularity in $k$ in the strong coupling limit (we restrict ourselves to the case $r=1$ ). We use the hopping parameter expansion and the random walk method [8] to derive an expansion for the meson propagators. The meson propagator corresponds to a random walk of quark-antiquark lines with double projection operators on the lattice.

We denote as $N_{L}(n, \alpha, \beta)$ of the number of possible random walks from the origin arriving at a site $n$ after $L$ steps $(\alpha, \beta$ are spin index of quark and antiquark). The possible numbers for $L$ steps and $L-1$ steps are related by a neighboring constraints for the lattice sites $n$ and $n^{\prime}$, which is characterized by a matrix

$N_{L}(n, \alpha, \beta)=\sum_{n^{\prime} \alpha^{\prime} \beta^{\prime}} M\left(n, \alpha, \beta, n^{\prime}, \alpha^{\prime}, \beta^{\prime}\right) N_{L}\left(n^{\prime}, \alpha^{\prime}, \beta^{\prime}\right)$

where

$$
\begin{aligned}
& M\left(n, \alpha, \beta, n^{\prime}, \alpha^{\prime}, \beta^{\prime}\right)=(2 k)^{2} \sum_{\mu}\left[\left(P_{\mu}^{-}\right)_{\alpha \alpha^{\prime}}\left(P_{\mu}^{+\mathrm{T}}\right)_{\beta \beta^{\prime}} \delta_{n+\mu, n^{\prime}}+\left(P_{\mu}^{+}\right)_{\alpha \alpha^{\prime}}\left(P_{\mu}^{-\mathrm{T}}\right)_{\beta \beta^{\prime}} \delta_{n-\mu, n^{\prime}}\right. \\
& \left.\quad+\left(Q_{\mu}^{-}\right)_{\alpha \alpha^{\prime}}\left(Q_{\mu}^{+\mathrm{T}}\right)_{\beta \beta^{\prime}} \delta_{n+2 \mu, n^{\prime}}+\left(Q_{\mu}^{+}\right)_{\alpha \alpha^{\prime}}\left(Q_{\mu}^{-\mathrm{T}}\right)_{\beta \beta^{\prime}} \delta_{n-2 \mu, n^{\prime}}\right]
\end{aligned}
$$

with

$P_{\mu}^{ \pm}=\frac{1}{2}\left(1 \pm \gamma_{\mu}\right), \quad Q_{\mu}^{ \pm}=-\frac{1}{8}\left(1 \pm \gamma_{\mu}\right)$,

$P_{\mu}^{ \pm}$and $Q_{\mu}^{ \pm}$correspond to the nearest neighbor and next nearest neighbor interaction respectively. $\mathrm{T}$ stands for the transpose of the matrix. Thus, if we consider the Green function of the quark-antiquark density operators for finite lattice spacing

$G_{\alpha \delta}^{\beta \gamma}(n)=\left\langle 0\left|\bar{\Psi}_{\gamma}(n) \Psi_{\alpha}(n) \bar{\Psi}_{\delta}(0) \Psi_{\beta}(0)\right| 0\right\rangle$

we notice that it can be rewritten as [8]

$G_{\alpha \delta}^{\beta \gamma}(n)=-N \int_{P} \mathrm{e}^{\mathrm{i} p n} D_{\alpha \delta}^{-1 \beta \gamma}(p)$,

with

$$
\begin{aligned}
& D_{\alpha \delta}^{\beta \gamma}=\delta_{\alpha}^{\beta} \delta_{\delta}^{\gamma}-(2 k)^{2} \sum_{\mu}\left[\left(P_{\mu}^{-}\right)_{\alpha}^{\beta}\left(P_{\mu}^{+}\right)_{\delta}^{\gamma} \exp \left(\mathrm{i}_{\mu}\right)+\left(P_{\mu}^{+}\right)_{\alpha}^{\beta}\left(P_{\mu}^{-}\right)_{\delta}^{\gamma} \exp \left(-\mathrm{i} p_{\mu}\right)\right. \\
& \left.\quad+\left(Q_{\mu}^{-}\right)_{\alpha}^{\beta}\left(Q_{\mu}^{+}\right)_{\delta}^{\gamma} \exp \left(2 \mathrm{i} p_{\mu}\right)+\left(Q_{\mu}^{+}\right)_{\alpha}^{\beta}\left(Q_{\mu}^{-}\right)_{\delta}^{\gamma} \exp \left(-2 \mathrm{i} p_{\mu}\right)\right]
\end{aligned}
$$

By Fierz transformation we expand the 16 by 16 matrix $D_{\alpha \delta}^{\beta \gamma}$ into another 16 by 16 matrix $D_{A B}$

$$
D_{\alpha \delta}^{\beta \gamma}=\sum_{A B} D_{A B}\left(\Gamma_{A}\right)_{\alpha}^{\gamma}\left(\Gamma_{B}\right)_{\delta}^{\beta}
$$

The non-zero components of coefficient matrix $D_{A B}$ are diagonal sub matrices $1, P_{P A}$, and $D_{V T}$. The zero eigenvalue for $D_{A B}$ with a particle at rest $p_{\mu}=(0, \mathrm{i} M)$ should give the pole position of the propagator, the mass of mesons.

For $D_{P A}$, it is given by

$$
D_{P A(0)}=\left(\begin{array}{ll}
1-\frac{51}{4} k^{2}-4 k^{2} \operatorname{ch} M-\frac{1}{4} k^{2} \operatorname{ch} 2 M & 4 k^{2} \mathrm{i}\left(\operatorname{sh} M+\frac{1}{16} \operatorname{sh} 2 M\right) \\
-4 k^{2} \mathrm{i}\left(\operatorname{sh} M+\frac{1}{16} \operatorname{sh} 2 M\right) & 1-4 k^{2} \operatorname{ch} M-\frac{1}{4} k^{2} \operatorname{ch} 2 M
\end{array}\right) .
$$

For $D_{V T}$, there are three similar degenerate matrices 
$D_{V(i) T(0, i)}=\left(\begin{array}{ll}1-\frac{17}{2} k^{2}-4 k^{2} \operatorname{ch} M-\frac{1}{4} k^{2} \operatorname{ch} 2 M & 4 k^{2}(1 / \sqrt{2}) \mathrm{i}\left(\operatorname{sh} M+\frac{1}{16} \operatorname{sh} 2 M\right) \\ 4 k^{2}(1 / \sqrt{2}) \mathrm{i}\left(\operatorname{sh} M+\frac{1}{16} \operatorname{sh} 2 M\right) & \frac{1}{2}\left[1-\frac{17}{4} k^{2}-4 k^{2} \operatorname{ch} M-\frac{1}{4} k^{2} \operatorname{ch} 2 M\right]\end{array}\right)$.

The constraint $\left\|D_{P A(0)}\right\|=0$ gives the meson mass of the $D_{P A}$ sector, and $\left\|D_{V(i) T(0, i)}\right\|=0$ gives a threefold degenerate meson mass of the $D_{V T}$ sector. The vanishing of the meson mass gives the critical value of $k$ in the strong coupling. The lowest value of $k_{\mathrm{c}}$ comes from the $D_{P A}$ sector

$k_{\mathrm{c}}=\sqrt{\frac{1}{17}}=0.242535$.

Note that this value is very close to the critical $k$ for the ordinary Wilson action at strong coupling, which is about one quarter.

3. Relationship between quark masses on the lattice and in the continuum. Actually it is possible to write down a fermion action which approaches the continuum limit even more rapidly than the one in eqs. (2.9), (2.10). In order to do this, let us take the coefficient not only of the $\mathrm{O}\left(p^{2}\right)$ but also of the $\mathrm{O}\left(p^{3}\right)$ terms in the fermion propagator to be zero. Then from eq. (2.8) we get

$C=-\frac{1}{4} k r, \quad D=-\frac{1}{8} k$.

The fermion propagator and the vertices now become (expanding in $p$ )

$$
\begin{aligned}
& S_{\mathrm{F}}^{-1}(p)=1-2 k \sum_{\mu}\left[\left(r \cos p_{\mu}-\mathrm{i} \gamma_{\mu} \sin p_{\mu}\right)-\left(\frac{1}{4} r \cos 2 p_{\mu}-\frac{1}{8} \mathrm{i} \gamma_{\mu} \sin 2 p_{\mu}\right)\right] \\
& \quad=\frac{3}{2} k\left(\mathrm{i} \sum_{\mu} \gamma_{\mu} p_{\mu}+m+\mathrm{O}\left(p^{4}\right)\right), \\
& V_{\mu}^{(3)}(p, q)=\frac{3}{2} \mathrm{i} k g \gamma_{\mu}\left[1+\frac{1}{24}(p-q)_{\mu}^{2}+\mathrm{O}\left(p^{3}\right)\right] t^{a}, \\
& V_{\mu \nu}^{(4)}(p, q)=-\frac{1}{4} g^{2} k r\left[3(p+q)_{\mu}^{2}+k_{\mu}^{2}+l_{\mu}^{2}+\mathrm{O}\left(p^{3}\right)\right] \delta_{\mu \nu} t^{a} t^{b},
\end{aligned}
$$

with $m=(2 / 3 k)(1-6 k r)$.

As discussed in ref. [9] and references therein, the relation between the running quark mass $m_{\mathrm{f}}(a)$ on the lattice and the running quark mass $m(\mu)$ at a scale $\mu$ in the continuum is given by

$m_{\mathrm{f}}(\mu)=m_{\mathrm{f}}(a)\left\{1-\left(6 g^{2} / 16 \pi^{2}\right)\left[\left(N^{2}-1\right) / 2 N\right]\left(\ln a \mu-\ln C_{m}\right)\right\}$,

where $a$ is the lattice spacing, $N$ is the number of color, and

$C_{m}=\Lambda_{m} / \Lambda_{m}^{\text {latt }}$.

$\Lambda_{m}$ and $\Lambda_{m}^{\text {latt }}$ are scales which are introduced in the equations that govern the renormalization group behavior of

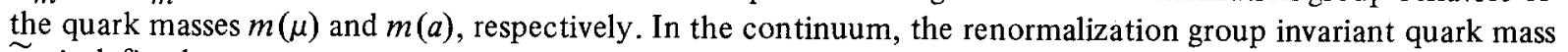
$\widetilde{m}_{\mathrm{f}}$ is defined as

$\widetilde{m}_{\mathrm{f}}=\left[\left(b_{0} / N\right) \ln (\mu / \Lambda)\right]^{-\gamma_{1} m / \beta_{0}} m(\mu)$,

$\Lambda$ is a mass parameter defined in some renormalization scheme. Also $b_{0}=11 N / 6 \pi$ and the quantities $\gamma_{1 m}$ and $\beta_{0}$ are the coefficients of the first term of $\gamma_{m}$ and $\beta$ function respectively. If, as a first approximation, the contribution of in ternal quark loop is neglected in a numerical simulation, we have

$\beta_{0}=11 N / 48 \pi^{2}$,

and 


$$
\begin{aligned}
\widetilde{m}_{\mathrm{f}} & =\left[\left(b_{0} / N\right) \ln \left(C_{m} / a \Lambda\right)\right]^{-\gamma_{1} m / \beta_{0}} m_{\mathrm{f}}(a) \\
& =\left[\left(2 \pi / N^{2}\right)\left(\beta+\left(N b_{0} / 2 \pi\right) \ln \left(\Lambda_{L A} / \Lambda\right)+\left(N b_{0} / 2 \pi\right) \ln C_{m}\right)\right]^{-\gamma_{1} m / \beta_{0}} m_{\mathrm{f}}(a),
\end{aligned}
$$

where $\Lambda_{L A}$ is the mass parameter defined on the lattice. By calculating the self energy of a quark perturbatively on the lattice and in the continuum we can extract the value of $C_{m}$. The general expression for the fermion self energy order $\mathrm{O}\left(g^{2}\right)$ for $q \rightarrow 0$ has the form

$$
\Sigma^{\mathrm{LATT}}(q)=\frac{3}{2} k\left(g^{2} / 16 \pi^{2}\right)\left[\left(N^{2}-1\right) / 2 N\right]\left[r \Sigma_{0}^{\mathrm{LATT}}+\mathrm{i} \phi \Sigma_{1}^{\mathrm{LATT}}+m \Sigma_{2}^{\mathrm{LATT}}\right] .
$$

As shown in the previous section, the effect of the $\Sigma_{0}^{\mathrm{LATT}}$ term is to shift the $k_{\mathrm{c}}\left(k_{\mathrm{c}}=1 / 6 r\right.$ at the tree level). From the diagrams of fig. 2(a) and (b), we obtain

$$
\begin{aligned}
& \Sigma_{0}^{\mathrm{LATT}}=-\frac{8 \pi^{2}}{3}+\frac{16 \pi^{2}}{9} \int_{p} \frac{1}{\Delta_{1} \Delta_{2}}\left(-\frac{8}{3} \sum_{\mu}\left(2 \cos \frac{1}{2} p_{\mu}-\frac{1}{2} \cos p_{\mu} \cos \frac{1}{2} p_{\mu}\right)^{2} \sum_{\rho} \sin ^{4} \frac{1}{2} p_{\rho}\right. \\
& \quad+\frac{8}{3} \sum_{\mu}\left(2 \sin \frac{1}{2} p_{\mu}-\sin p_{\mu} \cos \frac{1}{2} p_{\mu}\right)^{2} \sum_{\rho} \sin ^{4} \frac{1}{2} p_{\rho}+2 \sum_{\mu}\left(2 \cos \frac{1}{2} p_{\mu}-\frac{1}{2} \cos p_{\mu} \cos \frac{1}{2} p_{\mu}\right) \\
& \left.+\left(2 \sin \frac{1}{2} p_{\mu}-\sin p_{\mu} \cos \frac{1}{2} p_{\mu}\right) \sin p_{\mu}\left(1+\frac{2}{3} \sin ^{2} \frac{1}{2} p_{\mu}\right)\right) \\
& \Sigma_{1}^{\mathrm{LATT}}=2 \int_{0}^{1} x \mathrm{~d} x \ln \left[\left(q^{2} x+m^{2}\right)(1-x) a^{2}\right]+\gamma_{\mathrm{E}}-F_{0001}+\frac{2}{3} \pi^{2} \\
& \quad+\frac{4}{9} \pi^{2} \int_{p} \frac{1}{\Delta_{1} \Delta_{2}}\left(\frac{16}{3} r^{2} \sum_{\mu}\left(\frac{3}{2} \sin ^{2} \frac{1}{2} p_{\mu}-\sin ^{4} \frac{1}{2} p_{\mu}-2 \sin ^{6} \frac{1}{2} p_{\mu}\right) \sum_{\rho} \sin ^{4} \frac{1}{2} p_{\rho}\right. \\
& \left.\quad-\frac{3}{8} \sum_{\mu} \sin ^{2} p_{\mu}\left(1+\frac{2}{3} \sin ^{2} \frac{1}{2} p_{\mu}\right)^{2}\left(1+2 \sin ^{2} \frac{1}{2} p_{\mu}\right)+2 r^{2} \sum_{\mu} \sin ^{2} p_{\mu} \sin ^{4} \frac{1}{2} p_{\mu}\left(1+\frac{2}{3} \sin ^{2} \frac{1}{2} p_{\mu}\right)\right) \\
& \quad+\frac{4}{9} \pi^{2} \int_{p} \frac{r^{2}}{\Delta_{2} \Delta_{1}^{2}}\left(4 \sum_{\rho}^{1} \sin ^{4} \frac{1}{2} p_{\rho} \sum_{\mu} \sin ^{2} p_{\mu} \sin ^{2} \frac{1}{2} p_{\mu}\left(1+\frac{2}{3} \sin ^{2} \frac{1}{2} p_{\mu}\right)\right. \\
& \left.\quad-2 \sum_{\rho} \sin ^{6} \frac{1}{2} p_{\rho} \sum_{\mu} \sin ^{2} p_{\mu}\left(1+\frac{2}{3} \sin ^{2} \frac{1}{2} p_{\mu}\right)\right) \\
& \quad+\frac{4}{9} \pi^{2} \int_{p}\left[\frac { 1 } { \Delta _ { 2 } \Delta _ { 1 } ^ { 2 } } \left(\frac{9}{4} \sum_{\mu} \cos ^{2} \frac{1}{2} p_{\mu} \sin ^{2} p_{\mu}\left(1+\frac{2}{3} \sin ^{2} \frac{1}{2} p_{\mu}\right)^{3}\right.\right. \\
& \left.\left.\quad-\frac{9}{8} \sum_{\mu} \cos ^{2} \frac{1}{2} p_{\mu}\left(1+\frac{2}{3} \sin ^{2} \frac{1}{2} p_{\mu}\right)^{2} \sum_{\rho} \sin ^{2} p_{\rho}\left(1+\frac{2}{3} \sin ^{2} p_{\rho}\right)\right)+\frac{1}{\Delta_{1}^{3}} \frac{9}{16} \sum_{\mu} \sin ^{2} p_{\mu}\right] \\
&
\end{aligned}
$$

Table 1.

Some values of $\ln C_{m}$.

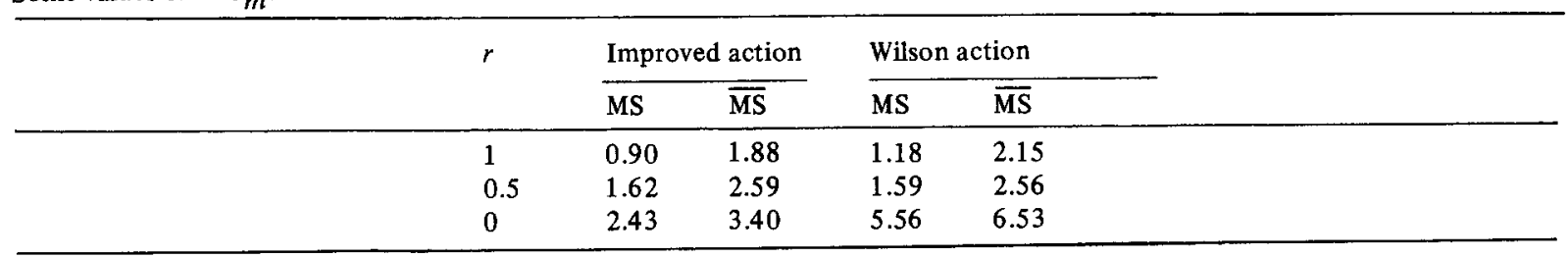




$$
\begin{aligned}
& \Sigma_{2}^{\mathrm{LATT}}=4 \int_{0}^{1} \mathrm{~d} x \ln \left[\left(q^{2} x+m^{2}\right)(1-x) a^{2}\right]+4 \gamma_{\mathrm{E}}-4 F_{0000} \\
& -\frac{256}{27} \pi^{2} r^{2} \int_{p} \frac{1}{\Delta_{1} \Delta_{2}^{2}} \sum_{\nu} \sin ^{4} \frac{1}{2} p_{\nu}\left(-6 \sum_{\mu} \cos ^{2} \frac{1}{2} p_{\mu}\left(1+\frac{2}{3} \sin ^{2} \frac{1}{2} p_{\mu}\right)^{2} \sum_{\rho} \sin ^{4} \frac{1}{2} p_{\rho}\right. \\
& \left.+\frac{32}{3} r^{2} \sum_{\mu} \sin ^{6} \frac{1}{2} p_{\mu} \sum_{\rho} \sin ^{4} \frac{1}{2} p_{\rho}+3 \sum_{\mu} \sin ^{2} p_{\mu} \sin ^{2} \frac{1}{2} p_{\mu}\left(1+\frac{2}{3} \sin ^{2} \frac{1}{2} p_{\mu}\right)^{2}\right) \\
& +4 \pi^{2} \int_{p}\left[\frac{1}{\Delta_{1} \Delta_{2}}\left(-\sum_{\mu}\left(1+\frac{2}{3} \sin ^{2} \frac{1}{2} p_{\mu}\right)^{2} \cos ^{2} \frac{1}{2} p_{\mu}+\frac{16}{9} r^{2} \sum_{\mu} \sin ^{6} \frac{1}{2} p_{\mu}\right)+\frac{1}{\Delta_{1}^{2}}\right],
\end{aligned}
$$

where $F_{0000}, F_{0001}$ are defined in ref. [10] $\gamma_{\mathrm{E}}$ is Euler's constant and

$\Delta_{1}=\sum_{\mu} \sin ^{2} \frac{1}{2} p_{\mu}, \quad \Delta_{2}=\left(\frac{8}{3} r \sum_{\mu} \sin ^{4} \frac{1}{2} p_{\mu}\right)^{2}+\sum_{\mu}\left[\sin p_{\mu}\left(1+\frac{2}{3} \sin ^{2} \frac{1}{2} p_{\mu}\right)\right]^{2}$.

On the other hand using dimensional regularization in the continuum theory one obtains (we use the MS scheme)

$\Sigma_{1}=2 \int_{0}^{1} x \mathrm{~d} x \ln \left[(1-x)\left(q^{2} x+m^{2}\right) / \mu^{2}\right]+\gamma_{\mathrm{E}}-\ln 4 \pi+1$,

$\Sigma_{2}=4 \int_{0}^{1} \mathrm{~d} x \ln \left[(1-x)\left(q^{2} x+m^{2}\right) / \mu^{2}\right]+4 \gamma_{\mathrm{E}}-4 \ln 4 \pi+2$

Then from eqs. (3.3), (3.8) and (3.13) we get

$$
C_{m}=\exp \frac{1}{6}\left[\left(\widetilde{\Sigma}_{1}^{\mathrm{LATT}}-\widetilde{\Sigma}_{2}^{\mathrm{LATT}}\right)-\left(\widetilde{\Sigma}_{1}-\widetilde{\Sigma}_{2}\right)\right]
$$

where " $\sim$ " stands for the finite part of corresponding quantities. $C_{m}$ has been numerically evaluated with an accuracy of better than $1 \%$ using gaussian quadrature formulae. In table 1 we give some numerical values of $\ln C_{m}$ in the MS and $\overline{\mathrm{MS}}$ schemes. The results in the $\overline{\mathrm{MS}}$ scheme is obtained by dropping the terms proportional to $\gamma_{\mathrm{E}}-\ln 4 \pi$ in $\Sigma_{1}$ and $\Sigma_{2}$.

At this point we would like to correct some slight errors which appear in ref. [9]. Using the Wilson action, they compute the relation between the quark masses on the lattice and in the continuum. In their formulation, $\Sigma_{1}$ and $\Sigma_{2}$ should be

$$
\begin{aligned}
& \widetilde{\Sigma}_{1}^{\mathrm{LATT}}=\gamma_{\mathrm{E}}-F_{0001}+\frac{1}{4 \pi^{2}} \int_{-\pi}^{\pi} \mathrm{d}^{4} p\left(\frac{r^{2}}{2} \frac{\Sigma_{\mu} \cos p_{\mu}}{\Delta_{2}}-\frac{1}{4} \frac{\Sigma_{\mu} \sin ^{2} \frac{1}{2} p_{\mu}-\sin ^{2} p_{\mu}}{\Delta_{2} \Delta_{1}^{2}}\right. \\
& \left.-\frac{1}{4} \frac{\Sigma_{\rho} \sin ^{2} p_{\rho}\left[\Sigma_{\mu} \sin ^{4} \frac{1}{2} p_{\mu}-r^{2}\left(\Sigma_{\mu} \sin ^{2} \frac{1}{2} p_{\mu}\right)^{2}\right]}{\Delta_{2} \Delta_{1}^{3}}+\frac{1}{2 \Delta_{1}}\right), \\
& \widetilde{\Sigma}_{2}^{\mathrm{LATT}}=4 \gamma_{\mathrm{E}}-4 F_{0000}-\frac{r^{2}}{\pi^{2}} \int_{-\pi}^{\pi} \mathrm{d}^{4} p \frac{1}{\Delta_{2}^{2}}\left[\left(8 r^{2}-2\left(1+r^{2}\right) \sum_{\mu} \cos ^{2} \frac{1}{2} p_{\mu}\right) \sum_{\mu} \sin ^{2} \frac{1}{2} p_{\mu}+\sum_{\mu} \sin ^{2} p_{\mu}\right] \\
& +\frac{1}{4 \pi^{2}} \int_{-\pi}^{\pi} \mathrm{d}^{4} p\left(\frac{1+r^{2}}{\Delta_{2}}-\frac{4 \Sigma_{\rho} \sin ^{4} \frac{1}{2} p_{\mu}-4 r^{2}\left(\Sigma_{\rho} \sin ^{2} \frac{1}{2} p_{\rho}\right)^{2}}{\Delta_{2} \Delta_{1}^{2}}\right),
\end{aligned}
$$


where

$\Delta_{2}=4 r^{2}\left(\sum_{\rho} \sin ^{2} \frac{1}{2} p_{\rho}\right)^{2}+\sum_{\rho} \sin ^{2} p_{\rho}$

This means that $\widetilde{m}_{\mathrm{f}}$ should become (in the MS scheme)

$\tilde{m}_{\mathrm{f}}=m_{\mathrm{f}}(a)\left[\frac{2}{9} \pi(\beta-1.99+0.99)\right] \approx 1.57 m_{\mathrm{f}}(a), \quad$ at $\beta=6$,

instead of $2.06 m_{\mathrm{f}}(a)$.

4. Discussion. We have presented above some analytical results concerning an improved fermion action on the lattice. One might expect that an improved action of the type we have described will prove useful in the context of numerical studies of lattice QCD, perhaps in connection with the use of an improved action also for the gauge fields.

We have also performed some preliminary numerical simulations with the improved fermion action. The relaxation program to compute the fermion propagator runs in the improved case about a factor of four slower than in the ordinary (Wilson) case. Unfortunately it appears that on small lattices $(4 \times 4 \times 4 \times 8 \times 6 \times 6 \times 6 \times 12)$ the finite size effects even at infinite gauge coupling are more significant than in the Wilson fermion case. This situation is, on the other hand, not unexpected given the presence of next-nearest neighbor terms in the new action. A detailed numerical study using the improved action would therefore appear meaningful only on larger lattices. We hope to return to this question in a future publication.

This research was supported by the US Department of Energy under grant no. DE-AC02-76ER02220.

\section{References}

[1] H. Hamber, plenary talk presented VI Congress on Mathematical physics (Boulder, CO) IAS preprint (July 1983); and references therein.

[2] K.G. Wilson, Cargese lecture notes 1979, ed. G. t'Hooft (Plenum, New York, 1979);

K. Symanzik, in: Mathematical problems in theoretical physics, eds. R. Schrader et al. (Springer, Berlin, 1982), Lecture Notes. in Physics 153.

[3] K. Symanzik, DESY preprints 83-016, 83-026.

[4] G. Martinelli, G. Parisi and R. Petronzio, Phys. Lett. 114B (1982) 251.

[5] P. Weisz, Nucl. Phys. B212 (1983) 1.

[6] G. Curci, P. Menotti and G.P. Paffuti, Phys. Lett. 130B (1983) 205; S. Belforte, G. Curci, P. Menotti and G.P. Paffuti, Pisa University preprint IFUP-TH 83/6.

[7] K.G. Wilson, Phys. Rev. D10 (1974) 2445; in: New phenomena in subnuclear physics (Erice 1975), ed. A. Zichichi (Plenum New York, 1977).

[8] N. Kawamoto, Nucl. Phys. B190 [FS3] (1981) 617.

[9] A. Gonzalez-Arroyo, G. Martinelli and F.J. Yndurain, Phys. Lett. 117B (1982) 437.

[10] A. Gonzalez-Arroyo and C.P. Korthals Altes, Nucl. Phys. B205 [FS5] (1982) 46. 